\title{
A new experiment at J-PARC to measure the neutrino cross section ratio between water and plastic
}

\author{
N. Chikuma ${ }^{* a}$, A. Izmaylov ${ }^{a}$, F. Hosomi ${ }^{a}$, T. Koga ${ }^{a}$, M. Yokoyama $^{a}$, \\ M. Khabibullin ${ }^{b}$, A. Khotjantsev ${ }^{b}$, Y. Kudenko ${ }^{b}$, A. Mefodiev ${ }^{b}$, O. Mineev $^{b}$, \\ T. Ovsjannikova ${ }^{b}$, S. Suvorov ${ }^{b}$, N. Yershov $^{b}$, A. Bonnemaison $^{c}$, R. Cornat ${ }^{c}$, \\ O. Drapier ${ }^{c}$, O. Ferreira ${ }^{c}$, F. Gastaldi ${ }^{c}$, M.Gonin $^{c}$, Th. A. Mueller ${ }^{c}$, T. Hayashino ${ }^{d}$, \\ A. K. Ichikawa ${ }^{d}$, A. Minamino ${ }^{d}$, K. Nakamura ${ }^{d}$, T. Nakaya ${ }^{d}$, B. Quilain ${ }^{d}$,

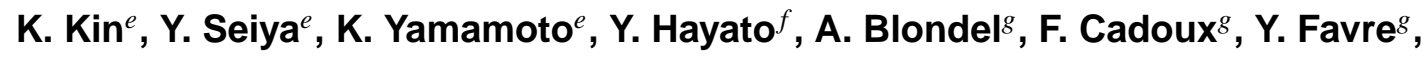 \\ E. Noah ${ }^{g}$, M. Rayner ${ }^{g}$ \\ ${ }^{a}$ The University of Tokyo, \\ ${ }^{b}$ Institute for Nuclear Research of the Russian Academy of Sciences, \\ ${ }^{c}$ Laboratoire Leprince-Ringuet, Ecole Polytechnique, \\ ${ }^{d}$ Kyoto University, \\ e Osaka City University, \\ ${ }^{f}$ Institute for Cosmic Ray Research, the University of Tokyo, \\ g Geneva University \\ E-mail: nchikuma@hep.phys.s.u-tokyo.ac.jp
}

\begin{abstract}
For precise measurement of neutrino oscillation, understanding of neutrino interactions on nucleus is indispensable. One of the main systematic errors in neutrino oscillation analysis in the T2K experiment is induced due to the difference in the acceptance and target materials between Super-Kamiokande, with water target, and the near detector, with plastic target. In order to reduce this uncertainty, a new experiment, named WAGASCI, at the neutrino beamline in Japan Proton Accelerator Research Complex (J-PARC) has been proposed. Its goal is to measure the cross section ratio of charged current neutrino interaction on nucleus between water target and plastic target with a few percent uncertainty. The detector adopts three-dimensional grid-like configuration of scintillator around water and plastic targets, to obtain large angular acceptance. Multi-pixel photon counters (MPPC) will be used to detect the light from scintillator. In this paper, the design of the new detector optimized with Monte Carlo simulation, R\&D of detector components, and the expected performance of the detector are reported.
\end{abstract}

Flavor Physics \& CP Violation 2015

May 25-29, 2015

Nagoya, Japan

\footnotetext{
* Speaker.
} 


\section{Introduction}

For the precise measurement of neutrino oscillation in $\mathrm{T} 2 \mathrm{~K}$, which is a long-baseline neutrino experiment with the neutrino beamline in Japan Proton Accelerator Research Complex (J-PARC), it is indispensable to understand better the neutrino charged current interaction with nucleus. The difference in the target materials between its far detector (water) and near detector (mainly plastic scintillator) induces the dominant part of the systematic uncertainty in the oscillation analysis [1]. The total systematic uncertainty for $v_{e}$ appearance is $6.8 \%$, of which $4.7 \%$ is due to the uncertainty of neutrino cross section in the far detector, that is not constrained by the measurement in the near detector. The uncertainty is also due to the difference in acceptance, because the far detector has $4 \pi$ acceptance to a charged particle from neutrino interaction whereas the near detector is mainly sensitive to forward scattering.

In order to reduce these uncertainties, a new experiment at the J-PARC neutrino beamline, named WAGASCI (WAter Grid And SCIntillator detector), is proposed to measure the charged current neutrino cross section ratio between water and plastic with large angular acceptance. The goals of this project are the followings; i) to measure the charged current cross section ratio between water and plastic with $3 \%$ uncertainty, ii) to measure the charge current cross section on water and plastic individually with large angular acceptance. Uncertainty of neutrino flux leads to the large uncertainty in measurement of absolute cross section. Taking the ratio of cross section by using the same neutrino flux, however, this uncertainty could be canceled and the precise measurement would be achieved [2].

\section{The WAGASCI experiment}

\subsection{Experimental configuration}

The WAGASCI detector will be located in the T2K neutrino near detector hall at J-PARC. Protons are accelerated by the synchrotron up to $30 \mathrm{GeV}$ and collide with a graphite target to produce charged pions. The pions are focused by three magnetic horns and decay mainly into muons and muon-neutrinos in the 96-m decay volume. The location for the detector will be on the $1.6^{\circ}$ off-axis from the beam center, where we expect a similar neutrino energy spectrum to that of $\mathrm{T} 2 \mathrm{~K}$, at $2.5^{\circ}$ off-axis. The neutrino energy at the peak of the spectrum is around $600 \mathrm{MeV}$.

\subsection{Detector design}

Figure 1 shows the overview of the WAGASCI detector. The detector consists of two parts. The first part, located at the center of the detector, consists of neutrino interaction targets, 1 ton of water and 1 ton of hydrocarbon, and 5120 plastic scintillator bars, and its total dimension is $1 \times 1 \times 2 \mathrm{~m}^{3}$. The water and hydrocarbon targets are arranged alternately to reduce the difference in the neutrino flux and the detection efficiency between targets. The thickness of scintillator bars is $3 \mathrm{~mm}$ and they form the three-dimensional grid-like structure as expressed in Fig. 2 This structure makes the detector sensitive to particles scattered at a large angle, and maximize the fraction of the target material to be $80 \%$ of the central detector.

The other part, located at right and left sides of the central detector and at the downstream, is 
the Muon Range Detectors (MRDs). The MRDs consist of 3 or 6-cm-thick iron plates and 7-mmthick plastic scintillator bars, and are structured as sandwich-like configuration. Neutrino events are identified by reconstructing tracks of charged particles from neutrino interaction in the central detector and identifying a muon from charged current interaction by the number of iron planes penetrated in MRDs. One of major background is due to particles generated in interaction outside the detector or in MRD which enter to the central detector. Such external background events are rejected by measuring the timing difference between the central detector and MRDs. The mass of iron is around 14 ton (49 ton) in the side (downstream) MRD, and the total thickness of iron is $30 \mathrm{~cm}(90 \mathrm{~cm})$.

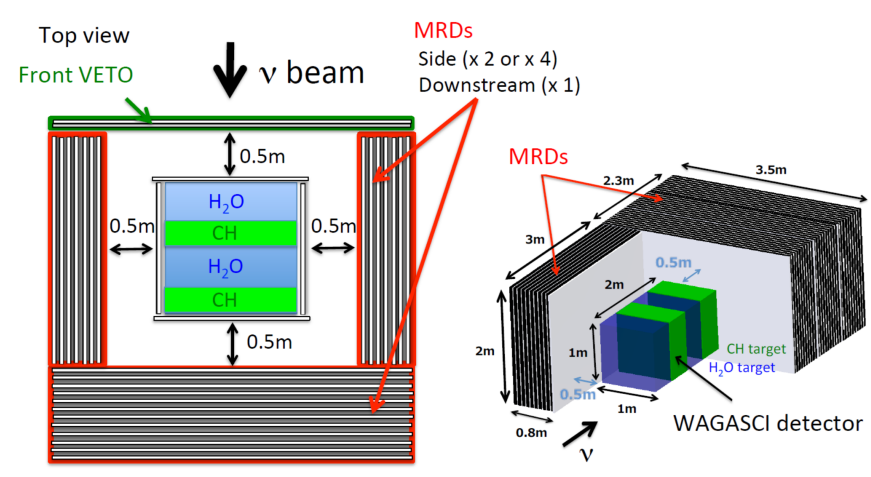

Figure 1: The configuration of the WAGASCI detectors.

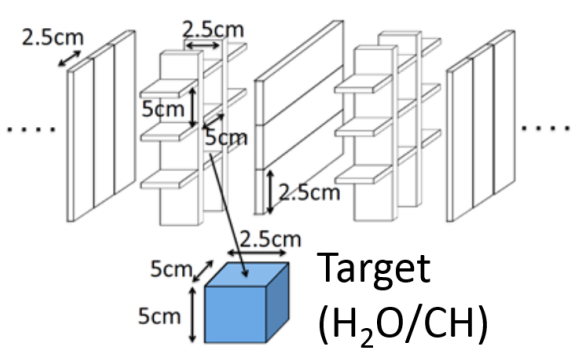

Figure 2: The three-dimensional grid-like structure of the plastic scintillator inside of the central detector.

\subsection{Expected performance}

The performance of the detector is evaluated by Monte Carlo simulation. As the event selection for the cross section measurement, the tracks of charged particles are required to penetrate 3(15)cm-thick iron plates in side (downstream) MRDs, in order to reduce the background from other charged particles than muons. Table 1 shows the expected number of events from the 1-ton water target with $10^{21}$ Protons On Target (POT). The number of charged current interactions after the event selection is expected to be $3 \times 10^{4}$ events with $90.1 \%$ purity.

Table 1: The expected number of events from 1 ton water target with $10^{21} \mathrm{POT}$.

\begin{tabular}{lllll}
\hline & $\begin{array}{l}\text { Charged current } \\
\text { interaction }\end{array}$ & $\begin{array}{l}\text { Neutral current } \\
\text { interaction }\end{array}$ & $\begin{array}{l}\text { Background } \\
\text { from outside }\end{array}$ & Total \\
\hline Events $\left[/ 10^{21} \mathrm{POT}\right]$ & $3.1 \times 10^{4}$ & $1.6 \times 10^{3}$ & $1.8 \times 10^{3}$ & $3.5 \times 10^{4}$ \\
Fraction & $90.1 \%$ & $4.6 \%$ & $5.2 \%$ & $100 \%$ \\
\hline
\end{tabular}

\section{R\&D of detector components}

\subsection{Performance test of $3 \mathrm{~mm}$ thin plastic scintillator bars}

The performance of 3-mm-thick plastic scintillator bars produced by Fermilab was measured at an electron synchrotron in Research Center for Electron Photon Science, Tohoku University. Using the $600-\mathrm{MeV}$ positron beam, the position dependencies of light yields and the detection efficiency are measured. The scintillation light is collected by a wavelength shifting (WLS) fiber 
to a photo detector, MPPC produced by Hamamatsu Photonics. The MPPC is operated with an overvoltage of $4.0 \mathrm{~V}$. The average light yields are ranged from 10 to 18 photoelectrons (p.e.), and the observed light yield decreases as the beam hit position is far from the WLS fiber. With a threshold of 1.5 p.e., the detection efficiency is better than $99 \%$ for the whole region of the scintillator bar.

\subsection{2-channel arrayed MPPC}

For the WAGASCI experiment, 32-channel arrayed MPPC are developed, which has low dark noise rate and after-pulsing rate, wider range of operation voltage $(\sim 5 \mathrm{~V})$, and low crosstalk rate. The mass production for WAGASCI will be done in the summer 2015 and their performance will be measured.

\section{Option to magnetize downstream MRD}

The anti-neutrino beam at J-PARC has a large contamination of neutrino background, and 30\% of interactions in the WAGASCI detector is expected to be interactions of neutrino background. Therefore, discrimination of anti-neutrino events from neutrino event is required. To achieve this purpose, an option to install an MRD with magnetized iron at the downstream of the central detector instead of the current downstream MRD is proposed. The sign of charge of particles from the neutrino interaction is identified by variation of the directions of their motion in the magnetic field.

The performance of charge identification is evaluated with Monte Carlo simulation. The efficiency to identify correctly the charge is expected to be $91 \%$, and the contamination of neutrino background would decrease down to $7 \%$ after the event selection. Optimization of the configuration of the downstream MRD is still going on, and the measurement of anti-neutrino cross section ratio between water and plastic with $3 \%$ uncertainty is expected to be achieved.

\section{Summary and schedule}

The WAGASCI experiment measures the water to plastic charged current cross section ratio with an accuracy of a few percent with large angular acceptance, by using the J-PARC neutrino beam. It will help to reduce the uncertainty of the neutrino oscillation measurement, such as the T2K experiment. The design of the detector and R\&D of the detector components are ongoing. The construction and installation of the WAGASCI detector will start at the end of 2015 and the physics operation will start at the autumn 2016.

\section{Acknowledgment}

This work was partially supported by JSPS KAKENHI and by the Russian Foundation for Basic Research Grant No.15-52-50015. The work of author (N.C.) has been supported by Advanced Leading Graduate Course for Photon Science (ALPS) at the University of Tokyo.

\section{References}

[1] K. Abe et al.(T2K Collaboration), Phys. Rev. D 91, 072010 (2015).

[2] K. Abe et al.(T2K Collaboration), Phys. Rev. D 90, 052010 (2014). 